\title{
A pegging approach to the precedence-constrained knapsack problem
}

\author{
Byungjun You ${ }^{1}$, Takeo Yamada * \\ Department of Computer Science, The National Defense Academy, Yokosuka, Kanagawa 239-8686, Japan
}

Received 6 May 2005; accepted 10 October 2006

Available online 13 December 2006

\begin{abstract}
The knapsack problem (KP) is generalized to the case where items are partially ordered through a set of precedence relations. As in ordinary KPs, each item is associated with profit and weight, the knapsack has a fixed capacity, and the problem is to determine the set of items to be packed in the knapsack. However, each item can be accepted only when all the preceding items have been included in the knapsack. The knapsack problem with these additional constraints is referred to as the precedence-constrained knapsack problem (PCKP). To solve PCKP exactly, we present a pegging approach, where the size of the original problem is reduced by applying the Lagrangian relaxation followed by a pegging test. Through this approach, we are able to solve PCKPs with thousands of items within a few minutes on an ordinary workstation.
\end{abstract}

(C) 2006 Elsevier B.V. All rights reserved.

Keywords: Combinational optimization; Pegging test; Knapsack problem; Precedence constraints

\section{Introduction}

Let $G=(V, E)$ be a directed graph $[10]$ with vertex set $V=\{1,2, \ldots, n\}$ and edge set $E \subseteq V \times V$. Here, V represents the set of items that can be included into a knapsack of capacity $c$. Associated with each $j \in V$ are its weight $w_{j}$ and profit $p_{j}$. Without much loss of generality we assume that parameters $w_{j}, p_{j}(j=1, \ldots, n)$ and $c$ are all positive integers. The set of edges $E$ represents precedence relations between the items. That is, $(i, j) \in E$ implies that item $j$ can be accepted only when item $i$ has been included in the knapsack. Throughout the paper, we put $m:=|E|$, and assume that $G$ is acyclic, i.e., no directed cycle is included in $G$, since otherwise the precedence relations are not well defined.

\footnotetext{
* Corresponding author.

E-mail address: yamada@nda.ac.jp (T. Yamada).

${ }^{1}$ Current address: The Republic of Korea Navy, Republic of Korea.
} 
The problem can be formulated mathematically as a $0-1$ programming problem. Let $x_{j}$ be a variable such that

$$
x_{j}= \begin{cases}1, & \text { if item } j \text { is accepted } \\ 0, & \text { otherwise }\end{cases}
$$

Then, we have the following precedence-constrained knapsack problem [21].

\section{PCKP:}

$$
\begin{array}{ll}
\text { maximize } & z(x):=\sum_{j=1}^{n} p_{j} x_{j} \\
\text { subject to } & \sum_{j=1}^{n} w_{j} x_{j} \leqslant c, \\
& x_{i} \geqslant x_{j}, \quad \forall(i, j) \in E, \\
& x_{j} \in\{0,1\}, \quad \forall j \in V .
\end{array}
$$

Here, without loss of generality, we assume that

$$
w_{j} \leqslant c \quad(\forall j \in V), \quad \sum_{j=1}^{n} w_{j}>c
$$

since otherwise the problem is trivial.

Precedence relations arise naturally as a consequence of logical/physical requirements among items. For example, in a project management activities are usually arranged in the form of a flow chart or a network, and each activity can be initiated only when all the preceding activities have been finished. Or, in open-pit mining [2] we can remove a block only when all the blocks lying immediately above have been removed. Mathematically, these relations are represented in the form of inequality (3). Then, if we wish to complete as many projects as possible, or excavate as many blocks as possible within a fixed time limit, we need to solve PCKP.

PCKP is $\mathscr{N} \mathscr{P}$-hard [9]; because without precedence constraint (3), it reduces to the knapsack problem (KP, $[17,15]$ ), which is already $\mathscr{N} \mathscr{P}$-hard. An important subclass of PCKP is the tree-knapsack problem (TKP, $[5,22,14,12])$, where $G$ is a directed tree rooted at node 1 . Hirabayashi et al. [11] formulated a tool-module design problem as a PCKP on a bipartite graph. Moriyama et al. [18] generalized this into a PCKP under the name of partially-ordered knapsack problem, and developed a branch-and-bound algorithm with some numerical experiments. Samphaiboon et al. [21] presented a dynamic programming algorithm to solve this problem. If the size of the problem is not so large, it may be solved by commercial or free IP solvers [8]. Using NUOPT [20], a popular IP solver in Japan, we were able to solve most of the randomly generated PCKPs with up to $n=2000$, but for larger problems we often encountered difficulties in obtaining exact solutions.

In this paper, we propose a novel approach to solve larger PCKPs exactly as follows. First, we eliminate constraints (3) by applying the Lagrangian relaxation, and together with the continuous relaxation of (4), the result is a continuous knapsack problem. Then, the pegging test for ordinary KPs can be applied, and if the Lagrangian multipliers are well tuned up, we obtain a PCKP of substantially reduced size. With the precedence constraint (3), we can further derive an improved block pegging test, and the reduced PCKPs are often solved by commercial IP solvers. We implement these algorithms, and evaluate the developed method through a series of computational experiments.

\section{Upper and lower bounds}

This section derives an upper bound by applying the Lagrangian relaxation $[19,23]$ to PCKP. We also present a local search [1] algorithm to obtain a good approximate solution quickly, which gives a lower bound to PCKP. 


\subsection{Lagrangian relaxation}

Given a nonnegative multiplier $\lambda:=\left(\lambda_{i j}\right) \in R^{m}$, the Lagrangian relaxation of PCKP is

$\operatorname{LPCKP}(\lambda)$ :

$$
\begin{array}{ll}
\operatorname{maximize} & L:=\sum_{j=1}^{n} p_{j} x_{j}+\sum_{(i, j) \in E} \lambda_{i j}\left(x_{i}-x_{j}\right) \\
\text { subject to } & \sum_{j=1}^{n} w_{j} x_{j} \leqslant c, \\
& 0 \leqslant x_{j} \leqslant 1, \quad \forall j \in V .
\end{array}
$$

Here $\lambda_{i j}$ is the Lagrangian multiplier associated with constraint (3). With 0-1 requirement (4) relaxed to continuous inequality (7), $\operatorname{LPCKP}(\lambda)$ is actually a continuous Lagrangian relaxation of PCKP.

The objective function (5) can be rewritten as

$$
L:=\sum_{j=1}^{n}\left(p_{j}+\sum_{k \in S_{j}^{+}} \lambda_{j k}-\sum_{i \in S_{j}^{-}} \lambda_{i j}\right) x_{j},
$$

where $S_{j}^{+}$(resp. $S_{j}^{-}$) is the set of terminating (resp. originating) vertices of edges that originate from (resp. terminate in) node $j$. If we fix $\lambda \geqslant 0, \operatorname{LPCKP}(\lambda)$ is the continuous $\mathrm{KP}$ whose solution is easily found [16]. Let $\bar{x}(\lambda)=\left(\bar{x}_{j}\right)$ denote an optimal solution to $\operatorname{LPCKP}(\lambda)$ with the corresponding optimal value $\bar{z}(\lambda)$, and $z^{\star}$ is the optimal objective value to PCKP. Then, we have

$$
z^{\star} \leqslant \bar{z}(\lambda)
$$

i.e., $\bar{z}(\lambda)$ gives an upper bound to PCKP. As a function of $\lambda$, it is known $[19,23]$ that

(i) $\bar{z}(\lambda)$ is a piecewise-linear, convex function of $\lambda$,

(ii) if $\bar{z}(\lambda)$ is differentiable at $\lambda$,

$$
\frac{\partial \bar{z}(\lambda)}{\partial \lambda_{i j}}=\bar{x}_{i}-\bar{x}_{j}, \quad \forall(i, j) \in E,
$$

(iii) for an arbitrary $\lambda \geqslant 0$, if $\bar{x}(\lambda)$ is feasible to PCKP and

$$
\lambda_{i j}\left(\bar{x}_{i}-\bar{x}_{j}\right)=0, \quad \forall(i, j) \in E,
$$

then $\bar{x}(\lambda)$ is an optimal solution to PCKP.

\subsection{Subgradient method}

For an arbitrary $\lambda \geqslant 0, \bar{z}(\lambda)$ gives an upper bound to PCKP. To make this value as small as possible, we employ the following subgradient method [19], where a subgradient is the vector $g=\left(g_{i j}\right)$ whose element is given by $(10)$ as $g_{i j}:=\bar{x}_{i}-\bar{x}_{j}$. Then, the direction of search $d=\left(d_{i j}\right)$ is the projection of $-g$ on the non-negative space, i.e.,

$$
d_{i j}:= \begin{cases}-g_{i j}, & \text { if } \lambda_{i j}>0 \text { or }\left(\lambda_{i j}=0 \text { and } g_{i j}<0\right), \\ 0, & \text { otherwise. }\end{cases}
$$

\section{Algorithm Subgradient_Method}

Step 1. Set $\lambda=0$.

Step 2. Solve $\operatorname{LPCKP}(\lambda)$.

Step 3. Calculate the subgradient $g$ and the direction of search $d$.

Step 4. (1-dim search) Find $\alpha^{\dagger} \geqslant 0$ such that $\bar{z}(\lambda+\alpha d)$ is minimized.

Step 5. If (11) is satisfied or $\bar{z}(\lambda) \cong \bar{z}\left(\lambda+\alpha^{\dagger} d\right)$, stop.

Step 6. Update $\lambda \leftarrow \lambda+\alpha^{\dagger} d$ and go to Step 2 . 
Let $\lambda^{\dagger}$ be $\lambda$ at the termination of the above algorithm. Then, we obtain the 'best' upper bound to PCKP as $\bar{z}:=\bar{z}\left(\lambda^{\dagger}\right)$.

\subsection{Lower bounds}

Let $\bar{x}\left(\lambda^{\dagger}\right)=\left(\bar{x}_{j}^{\dagger}\right)$ be an optimal solution to $\operatorname{LPCKP}\left(\lambda^{\dagger}\right)$. If this satisfies (3) and (4), this is a feasible solution; hence, the corresponding objective value gives a lower bound to PCKP. If some of the constraints (3) and (4) are violated, we still obtain a feasible solution by modifying $\bar{x}\left(\lambda^{\dagger}\right)$ in the following way: if $0<\bar{x}_{j}^{\dagger}<1$ for some $j \in V$ or if $\bar{x}_{j}^{\dagger}>\bar{x}_{i}^{\dagger}$ for some $(i, j) \in E$, we simply put $\bar{x}_{j}^{\dagger} \leftarrow 0$. We call the resulting feasible solution the Lagrangian solution to PCKP.

To further improve the solution, we employ the 2-opt method [1], which repeat the following as long as possible.

Procedure 2-opt

(i) In the current solution $x$ find a pair of items $i$ and $j$, such that $x_{i}=1$ and $x_{j}=0$, and furthermore these can be swapped as $x_{i}=0$ and $x_{j}=1$ without destroying feasibility of the resulting solution.

(ii) If this increases the value of the knapsack, carry out this swapping.

We call the resulting solution the 2-opt solution, with the corresponding lower bound denoted as $\underline{z}$.

\section{Pegging tests}

A pegging test is well known for the standard 0-1 KP $[13,7,6]$. By applying this test, many variables are fixed either at 0 or 1 , and removing these variables we obtain a problem of (often significantly) reduced size. In this section, we show that the same pegging test can be applied to PCKPs if we introduce the Lagrangian relaxation first.

\subsection{Plain pegging test}

Assume that we have an optimal Lagrangian multiplier $\lambda^{\dagger}$, the corresponding upper bound $\bar{z}=\bar{z}\left(\lambda^{\dagger}\right)$, and a lower bound $\underline{z}$ to PCKP, and let us define:

$$
\bar{p}_{j}:=p_{j}+\sum_{k \in S_{j}^{+}} \lambda_{j k}^{\dagger}-\sum_{i \in S_{j}^{-}} \lambda_{i j}^{\dagger} .
$$

Then, $\operatorname{LPCKP}\left(\lambda^{\dagger}\right)$ can be rewritten as

$$
\begin{array}{ll}
\text { maximize } & \sum_{j=1}^{n} \bar{p}_{j} x_{j} \\
\text { subject to } & (6) \text { and (7). }
\end{array}
$$

For an arbitrary $j=1, \ldots, n$, let $z^{\star}\left(x_{j}=u\right)$ denote the optimal objective value to PCKP with an additional constraint $x_{j}=u$, where $u$ is either 0 or 1 . Similarly, $\bar{z}\left(x_{j}=u\right)$ denotes the optimal objective value to $\operatorname{LPCKP}\left(\lambda^{\dagger}\right)$ with $x_{j}=u$. Then, for $j=1, \ldots, n$ the followings are obvious.

$$
\begin{aligned}
& z^{\star}=\max \left\{z^{\star}\left(x_{j}=0\right), \quad z^{\star}\left(x_{j}=1\right)\right\}, \\
& z^{\star}\left(x_{j}=u\right) \leqslant \bar{z}\left(x_{j}=u\right) .
\end{aligned}
$$

Then, if

$$
\bar{z}\left(x_{j}=0\right)<\underline{z},
$$


$x_{j}^{\star}=0$ is not possible in any optimal solution $x^{\star}=\left(x_{k}^{\star}\right)$ to PCKP, i.e., we necessarily have $x_{j}^{\star}=1$. Similarly, in the case that

$$
\bar{z}\left(x_{j}=1\right)<\underline{z},
$$

$x_{j}^{\star}=0$ must follow. This is the basic idea of the pegging test. To determine (17) and (18) quickly, the following shortcut is taken. First of all, without loss of generality, we assume the following.

$\mathbf{A}_{1}: \bar{p}_{j}>0, \quad \forall j \in V$,

$\mathbf{A}_{2}$ : The items are ordered in the non-increasing order of $\bar{p}_{j} / w_{j}$.

Let $W_{j}$ and $P_{j}$ be, respectively, the cumulative weight and profit, i.e.,

$$
W_{j}:=\sum_{i=1}^{j} w_{i}, \quad P_{j}:=\sum_{i=1}^{j} \bar{p}_{i},
$$

where $W_{0}=P_{0}=0$. Then, $\left\{\left(W_{j}, P_{j}\right) \mid j=0, \ldots, n\right\}$ gives a piecewise-linear, monotonically non-decreasing, concave function [16].

The intersection of this broken line with the vertical line $W=c$ gives the upper bound $\bar{z}$. The item $s$ satisfying $W_{s-1}<c \leqslant W_{s}$ is said to be a critical item, and we define

$$
r_{s}:=\bar{p}_{s} / w_{s} \text {. }
$$

Here, if for any $j<s$ we set $x_{j}=0$, it is known [13,7] that

$$
\bar{z}\left(x_{j}=0\right) \leqslant \bar{z}-\theta_{j},
$$

where we define

$$
\theta_{j}:=\bar{p}_{j}-r_{s} w_{j} .
$$

Then, if

$$
\bar{z}-\underline{z}<\theta_{j}
$$

from (20) we have $\bar{z}\left(x_{j}=0\right)<\underline{z}$, and thus $x_{j}^{\star}=1$. By a similar argument, if

$$
\bar{z}-\underline{z}<-\theta_{j}
$$

for any $j>s$, we obtain $x_{j}^{\star}=0$. Thus we have the following.

Theorem 1. For any optimal solution $x^{\star}=\left(x_{j}^{\star}\right)$ to PCKP, both of the followings are true.

(i) $\bar{z}-\underline{z}<\theta_{j} \Rightarrow x_{j}^{\star}=1$,

(ii) $\bar{z}-\underline{z}<-\theta_{j} \Rightarrow x_{j}^{\star}=0$.

Given a pair of upper and lower bounds, by applying this theorem some variables are fixed either at 0 or 1 , and removing these variables we obtain a PCKP of (often significantly) reduced size. We call this reduction by pegging test, and thus obtain a reduced problem.

\subsection{Block pegging test}

Again, we consider $\operatorname{LPCKP}\left(\lambda^{\dagger}\right)$, and assume $\mathbf{A}_{1}$ and $\mathbf{A}_{2}$ as before. Item $s$ stands for the critical item. In the acyclic graph $G=(V, E)$, if there exists a directed path from node $i$ to $j$, we say that $j$ is a descendent of $i$. By $D_{j}$ we denote the set of all descendents of $j$, where we note that this includes node $j$ itself. Furthermore, for an arbitrary node $j$, we define a subset of $D_{j}$ by

$$
D_{j}^{\prime}:=\left\{k \in D_{j} \mid k<s\right\} .
$$


Then, by setting $x_{j}=0$, we have $x_{k}=0$ for all $k \in D_{j}^{\prime}$, and the broken line connecting $\left(W_{0}, P_{0}\right)$, $\left(W_{1}, P_{1}\right), \ldots,\left(W_{n}, P_{n}\right)$ will be shifted to the lower leftwards by $\left(w_{j}^{\prime}, p_{j}^{\prime}\right)$, where we put

$$
w_{j}^{\prime}:=\sum_{k \in D_{j}^{\prime}} w_{k}, \quad \bar{p}_{j}^{\prime}:=\sum_{k \in D_{j}^{\prime}} \bar{p}_{k} .
$$

Define

$$
\Theta_{j}:=\bar{p}_{j}^{\prime}-r_{s} w_{j}^{\prime} .
$$

Then, similar to (22) if

$$
\bar{z}-\underline{z}<\Theta_{j}
$$

we necessarily obtain $x_{j}^{\star}=1$.

Similarly by considering the case of $j>s$, we obtain the following theorem.

Theorem 2. For any optimal solution $x^{\star}=\left(x_{j}^{\star}\right)$ to PCKP, both of the followings hold.

(i) $\bar{z}-\underline{z}<\Theta_{j} \Rightarrow x_{j}^{\star}=1$,

(ii) $\bar{z}-\underline{z}<-\Theta_{j} \Rightarrow x_{j}^{\star}=0$.

\subsection{Pegging algorithm}

Now we can solve PCKP in the following way.

\section{Algorithm Plain_Pegging (resp. Block_Pegging)}

Step 1. Compute the upper and lower bounds by the Lagrangian relaxation and 2-opt methods.

Step 2. Reduce the problem size by applying Theorem 1 (resp. Theorem 2).

Step 3. Solve the reduced problem using an appropriate IP solver.

Here, if we use the plain pegging test (Theorem 1) to reduce the problem size, we call this the plain pegging; and if Theorem 2 is used, we have the block pegging algorithm. Step 2 can be done by calculating $\theta_{j}$ (or $\Theta_{j}$, resp.) and checking if (22) (or (26), resp.) is satisfied for $j=1, \ldots, n$. This takes $O(n)$ time. After this, the reduced problem can be solved using a commercial or free IP solver.

\section{Virtual pegging test}

The usefulness of the pegging test depends on the gap between the upper and lower bounds. If the gap is not small enough, the effectiveness of our method is limited, since the size of the problem will not be reduced much in such a case. In this section, we introduce the virtual pegging test to cope with this problem.

\subsection{The principle}

In the pegging test, we input a pair of upper and lower bounds $\bar{z}$ and $\underline{z}$ to the pegging algorithm, and partition the original problem into a fixed part and the remaining reduced problem. Here, upper and lower bounds satisfy

$$
\underline{z} \leqslant z^{\star} \leqslant \bar{z}
$$

However, we can try the pegging test using an arbitrary value $l$ within $[\underline{z}, \bar{z}]$ as a hypothetical lower bound. Such an $l$ is referred to as a trial value.

Let the set of all the feasible solutions $x=\left(x_{j}\right)$ satisfying (2)-(4) be $X$. Then, if we carry out the pegging test with $\bar{z}$ and $l$, some $x_{j}$ 's will be fixed either at 0 or 1 . But this pegging is not guaranteed to be correct because $l$ is not necessarily a lower bound. Let the index sets of the variables fixed at 0 (or 1 ) by the above pegging procedure be $F_{0}(l)\left(F_{1}(l)\right.$, resp.). Then, we have the following reduced problem. 


$$
\begin{array}{ll}
\mathbf{R}(l): & \\
\text { maximize } & \sum_{j=1}^{n} p_{j} x_{j} \\
\text { subject to } & x \in X, \\
& x_{j}=1, \quad j \in F_{1}(l), \\
& x_{j}=0, \quad j \in F_{0}(l) .
\end{array}
$$

The optimal objective value to this problem will be denoted as $z_{l}^{\star}$, and is referred to as the realization for the trial value $l$. If $\mathbf{R}(l)$ is infeasible, we define $z_{l}^{\star}:=-\infty$. Then, we have

Theorem 3. For an arbitrary trial value $l \leqslant \bar{z}$ and its realization $z_{l}^{\star}$, the followings are true.

(i) $l \leqslant z^{\star} \Rightarrow z_{l}^{\star}=z^{\star}$,

(ii) $l>z^{\star} \Rightarrow z_{l}^{\star} \leqslant z^{\star}$

(iii) $l \leqslant l^{\prime} \Rightarrow z_{l}^{\star} \geqslant z_{l^{\prime}}^{\star}$

(iv) $l \leqslant z_{l}^{\star} \Rightarrow z_{l}^{\star}=z^{\star}$.

Proof. (i) If $l \leqslant z^{\star}, l$ is actually a lower bound; thus the pegging test works correctly and finds the optimal value $z^{\star}$, i.e., $z_{l}^{\star}=z^{\star}$. (ii) Note that for an arbitrary $l \leqslant \bar{z}, \mathbf{R}(l)$ is PCKP with the additional constraints (30) and (31). Then, by definition, the optimal objective values satisfy this relation. (iii) In applying Theorem 1 (or Theorem 2) with the gap of $\bar{z}-l$, we note that the larger the trial value $l$ is (and thus the smaller the gap is), the more variables are fixed, i.e., for $l \leqslant l^{\prime}$ we have $F_{u}(l) \subseteq F_{u}\left(l^{\prime}\right)(u=0,1)$. From this, the above relation is straightforward. (iv) If $l>z^{\star}$, from (ii) we have $z_{l}^{\star} \leqslant z^{\star}$, and thus $l>z_{l}^{\star}$. This is a contradiction. So, we have $l \leqslant z^{\star}$, and from (i) obtain $z_{l}^{\star}=z^{\star}$.

As a direct corollary of (iii), if $R(l)$ is infeasible, then $\mathbf{R}\left(l^{\prime}\right)$ is also infeasible for all $l^{\prime} \geqslant l$.

\subsection{A virtual pegging algorithm}

For an arbitrary trial value $l$, after carrying out the virtual pegging test and solving the reduced problem $\mathbf{R}(l)$, we obtain the corresponding $z_{l}^{\star}$. Then, if (iv) is satisfied in Theorem 3, the problem is solved. In addition, if gap $:=\bar{z}-l$ is small, it is probable that $\mathbf{R}(l)$ is a much smaller problem than the original. Thus, in such a case we obtain an optimal solution by solving this reduced problem. We propose the following algorithm, which includes measures for the case where (iv) is not satisfied.

\section{Algorithm Virtual_Pegging_Test}

Step 1. $l \leftarrow \max \{\bar{z}-\delta, \underline{z}\}$.

Step 2. Carry out the pegging test with trial value $l$, solve $\mathbf{R}(l)$ and obtain $z_{l}^{\star}$.

Step 3. If $l \leqslant z_{l}^{\star}$, go to Step 5 .

Step 4. Update $\underline{z} \leftarrow \max \left\{\underline{z}, z_{l}^{\star}\right\}$ and $l \leftarrow \max \{l-\delta, \underline{z}\}$, and go to Step 2 .

Step 5. The optimal value is obtained as $z^{\star}=z_{l}^{\star}$.

Here, $\delta$ is an arbitrary 'small' margin. We set the trial value initially at $l=\bar{z}-\delta$ unless this is not smaller than $\underline{z}$. Then, if the optimal value is not found in step 3, the trial value is further lowered by $\delta$, and we repeat Steps 2-4 all over again until an optimal solution is found.

\section{Numerical experiments}

In this section we evaluate the performance of our approach through a series of numerical experiments. We implemented the algorithm in C language and conducted some computation on an IBM RS/6000 Model 270 
workstation (CPU: POWER3-II SMP 2way, $375 \mathrm{MHz}$ ). The program (including a subprogram to generate random instances) is available from [24] with some explanatory materials.

\subsection{Test problems}

The number of items $n$ is set between 1000 and 16,000, and the weights and profits are assumed as the following.

- Uncorrelated case (UNCOR)

$w_{j}$ : Uniformly random over $[1,1000]$,

$p_{j}$ : Uniformly random over $[1,1000]$, independent of $w_{j}$.

- Weakly correlated case (WEAK)

$\left[w_{j}\right]$ : Uniformly random over $[1,1000]$,

$\left[p_{j}\right]$ : Uniformly random over $\left[w_{j}, w_{j}+200\right]$.

The capacity of the knapsack is fixed at first at $c=250 n$. This means that without precedence constraints approximately a half of all the items can be included in the knapsack, since the average weight of items is approximately 500. The precedence constraints are generated randomly with probability $d /(n-1)$. Hence, the average number of precedence constraints is $n d / 2$, where parameter $d$ controls the density of this constraints, and we examined the cases of $d=0.1-0.8$.

\subsection{Upper and lower bounds}

Tables 1 and 2 give an overview of the computation of upper and lower bounds for the UNCOR and WEAK cases, respectively. Here shown are $d, n, m$, the gap between the Lagrangian upper bound $\bar{z}$ and the 2-opt lower bound $\underline{z}$, as well as the computing time in seconds. The column 'ratio' shows the percentage

Table 1

Upper and lower bounds (UNCOR)

\begin{tabular}{|c|c|c|c|c|c|c|c|c|c|}
\hline \multirow[t]{2}{*}{$d$} & \multirow[t]{2}{*}{$n$} & \multirow[t]{2}{*}{$m$} & \multicolumn{3}{|l|}{ Gap } & \multicolumn{4}{|c|}{ CPU seconds } \\
\hline & & & Average & Maximum & Minimum & Average & Maximum & Minimum & Ratio \\
\hline \multirow[t]{5}{*}{0.1} & 1000 & 55.2 & 118.3 & 856 & 10 & 0.22 & 0.37 & 0.13 & 0.86 \\
\hline & 2000 & 100.3 & 35.4 & 65 & 3 & 0.70 & 1.01 & 0.52 & 0.73 \\
\hline & 4000 & 198.6 & 37.9 & 58 & 18 & 2.62 & 3.14 & 1.91 & 0.71 \\
\hline & 8000 & 402.4 & 25.6 & 54 & 8 & 8.21 & 9.69 & 6.79 & 0.57 \\
\hline & 16,000 & 807.4 & 31.5 & 66 & 16 & 29.89 & 36.77 & 24.57 & 0.46 \\
\hline \multirow[t]{5}{*}{0.2} & 1000 & 107.4 & 59.5 & 114 & 19 & 0.34 & 0.19 & 0.67 & 0.88 \\
\hline & 2000 & 201.2 & 37.0 & 63 & 9 & 1.00 & 2.28 & 0.64 & 0.82 \\
\hline & 4000 & 395.8 & 47.3 & 80 & 17 & 3.14 & 5.04 & 1.99 & 0.74 \\
\hline & 8000 & 800.2 & 23.7 & 67 & 4 & 11.79 & 21.16 & 6.04 & 0.73 \\
\hline & 16,000 & 1609.1 & 24.5 & 43 & 3 & 40.18 & 63.18 & 29.81 & 0.64 \\
\hline \multirow[t]{5}{*}{0.4} & 1000 & 206.2 & 85.3 & 189 & 43 & 0.61 & 1.32 & 0.33 & 0.93 \\
\hline & 2000 & 402.9 & 129.7 & 542 & 4 & 2.11 & 4.12 & 0.86 & 0.92 \\
\hline & 4000 & 807.1 & 111.3 & 691 & 21 & 8.35 & 16.82 & 3.97 & 0.90 \\
\hline & 8000 & 1593.0 & 160.4 & 1131 & 12 & 24.14 & 26.68 & 17.47 & 0.85 \\
\hline & 16,000 & 3210.3 & 78.6 & 253 & 3 & 82.95 & 108.95 & 61.95 & 0.83 \\
\hline \multirow[t]{5}{*}{0.8} & 1000 & 406.0 & 206.8 & 618 & 21 & 1.46 & 2.79 & 0.58 & 0.97 \\
\hline & 2000 & 802.3 & 349.5 & 1051 & 37 & 6.65 & 13.68 & 3.41 & 0.97 \\
\hline & 4000 & 1606.3 & 349.1 & 1925 & 22 & 26.93 & 35.52 & 18.35 & 0.97 \\
\hline & 8000 & 3196.7 & 227.6 & 1021 & 12 & 76.28 & 126.77 & 52.19 & 0.95 \\
\hline & 16,000 & 6402.6 & 340.9 & 1036 & 19 & 357.58 & 466.54 & 235.50 & 0.95 \\
\hline
\end{tabular}


Table 2

Upper and lower bounds (WEAK)

\begin{tabular}{|c|c|c|c|c|c|c|c|c|c|}
\hline \multirow[t]{2}{*}{$d$} & \multirow[t]{2}{*}{$n$} & \multirow[t]{2}{*}{$m$} & \multicolumn{3}{|l|}{ Gap } & \multicolumn{4}{|c|}{ CPU seconds } \\
\hline & & & Average & Maximum & Minimum & Average & Maximum & Minimum & Ratio \\
\hline \multirow[t]{5}{*}{0.1} & 1000 & 55.2 & 21.9 & 42 & 7 & 0.21 & 0.33 & 0.13 & 0.80 \\
\hline & 2000 & 100.3 & 18.2 & 54 & 5 & 0.61 & 0.94 & 0.43 & 0.70 \\
\hline & 4000 & 198.6 & 13.6 & 41 & 4 & 1.89 & 2.75 & 1.38 & 0.65 \\
\hline & 8000 & 402.4 & 36.4 & 99 & 3 & 7.88 & 10.17 & 6.50 & 0.46 \\
\hline & 16,000 & 807.4 & 40.5 & 107 & 10 & 31.57 & 49.24 & 24.07 & 0.39 \\
\hline \multirow[t]{5}{*}{0.2} & 1000 & 107.4 & 28.7 & 73 & 11 & 0.25 & 0.39 & 0.19 & 0.84 \\
\hline & 2000 & 201.2 & 33.7 & 103 & 8 & 0.92 & 1.64 & 0.46 & 0.79 \\
\hline & 4000 & 395.8 & 20.7 & 35 & 6 & 2.78 & 4.06 & 1.68 & 0.72 \\
\hline & 8000 & 800.2 & 64.9 & 268 & 8 & 11.93 & 19.33 & 6.95 & 0.67 \\
\hline & 16,000 & 1609.1 & 66.7 & 257 & 6 & 36.83 & 46.81 & 29.06 & 0.54 \\
\hline \multirow[t]{5}{*}{0.4} & 1000 & 206.2 & 36.3 & 110 & 11 & 0.50 & 1.09 & 0.23 & 0.91 \\
\hline & 2000 & 402.9 & 34.0 & 92 & 2 & 1.31 & 2.55 & 0.71 & 0.87 \\
\hline & 4000 & 807.1 & 70.0 & 271 & 2 & 6.40 & 10.00 & 2.78 & 0.87 \\
\hline & 8000 & 1593.0 & 73.6 & 198 & 11 & 22.86 & 39.52 & 15.04 & 0.83 \\
\hline & 16,000 & 3210.3 & 58.2 & 192 & 3 & 75.91 & 95.95 & 63.19 & 0.79 \\
\hline \multirow[t]{5}{*}{0.8} & 1000 & 406.0 & 54.8 & 135 & 18 & 1.17 & 1.64 & 0.43 & 0.98 \\
\hline & 2000 & 802.3 & 79.2 & 310 & 12 & 4.65 & 7.38 & 2.54 & 0.97 \\
\hline & 4000 & 1606.3 & 116.1 & 252 & 5 & 14.90 & 25.75 & 9.98 & 0.97 \\
\hline & 8000 & 3196.7 & 111.6 & 360 & 6 & 56.85 & 84.53 & 36.48 & 0.94 \\
\hline & 16,000 & 6402.6 & 358.2 & 914 & 69 & 292.16 & 530.76 & 136.33 & 0.93 \\
\hline
\end{tabular}

of the CPU time spent for the computation of the upper bound. In these tables, each row shows the average, maximum and minimum of these values over 10 randomly generated instances.

From these tables, we observe the followings:

1. The gap between $\bar{z}$ and $\underline{z}$ usually increases with $d$, and it is often smaller in WEAK than in UNCOR.

2. Computation time for the upper bound increases with $n$ as well as with $d$, but it is rather insensitive to the correlation type of the instances. CPU time to compute the lower bounds also increases with $n$, but it stays almost constant with the increase of $d$ or the change of correlation type. As a consequence, with the increase of $d$ the CPU time to compute the upper bounds becomes relatively time consuming (see the column of 'ratio').

\subsection{Problem reduction by pegging tests}

Tables 3 and 4 summarize the results of computation of the pegging tests. Here the columns PLAIN and BLOCK show the results of plain and block pegging, respectively. The size of the reduced problem is shown by $n^{\prime}$ (the number of variables) and $m^{\prime}$ (the number of precedence constraints), and the ratio of reduction is given by 'reduc', which is defined as the 'geometric mean' of $n^{\prime} / n$ and $m^{\prime} / m$, i.e.,

$$
\text { reduc }:=\sqrt{n^{\prime} m^{\prime} / n m} \text {. }
$$

Computing time for the pegging tests is negligible; indeed, in all cases tested it took at most 0.03 seconds.

The findings from these tables are as follows

1. The reduced problem is always smaller (although slightly) in the block pegging method than in the plain pegging.

2. The pegging method becomes less effective as $d$ increases. Also, correlation between weights and profits makes the pegging less effective. 
Table 3

Pegging test (UNCOR)

\begin{tabular}{|c|c|c|c|c|c|c|c|}
\hline \multirow[t]{2}{*}{$\bar{d}$} & \multirow[t]{2}{*}{$n$} & \multicolumn{3}{|l|}{ PLAIN } & \multicolumn{3}{|c|}{ BLOCK } \\
\hline & & $n^{\prime}$ & $m^{\prime}$ & Reduction & $n^{\prime}$ & $m^{\prime}$ & Reduction \\
\hline \multirow[t]{5}{*}{0.1} & 1000 & 162.4 & 7.2 & 0.14 & 161.5 & 6.2 & 0.13 \\
\hline & 2000 & 145.5 & 3.9 & 0.05 & 144.3 & 2.7 & 0.04 \\
\hline & 4000 & 305.5 & 8.9 & 0.06 & 304.0 & 7.4 & 0.05 \\
\hline & 8000 & 421.1 & 12.0 & 0.04 & 417.9 & 8.7 & 0.03 \\
\hline & 16,000 & 1011.3 & 27.2 & 0.05 & 1003.4 & 19.1 & 0.04 \\
\hline \multirow[t]{5}{*}{0.2} & 1000 & 118.9 & 8.1 & 0.09 & 117.0 & 6.0 & 0.08 \\
\hline & 2000 & 152.2 & 5.7 & 0.05 & 150.7 & 4.1 & 0.04 \\
\hline & 4000 & 371.8 & 17.7 & 0.06 & 367.5 & 13.2 & 0.06 \\
\hline & 8000 & 396.5 & 20.1 & 0.04 & 392.6 & 16.1 & 0.03 \\
\hline & 16,000 & 811.3 & 38.3 & 0.03 & 801.9 & 28.9 & 0.03 \\
\hline \multirow[t]{5}{*}{0.4} & 1000 & 167.9 & 19.4 & 0.13 & 163.2 & 14.4 & 0.11 \\
\hline & 2000 & 436.6 & 68.8 & 0.19 & 422.7 & 52.4 & 0.17 \\
\hline & 4000 & 715.8 & 108.8 & 0.15 & 693.4 & 82.7 & 0.13 \\
\hline & 8000 & 1575.1 & 242.5 & 0.17 & 1543.9 & 205.8 & 0.16 \\
\hline & 16,000 & 2416.2 & 310.2 & 0.12 & 2335.4 & 217.0 & 0.10 \\
\hline \multirow[t]{5}{*}{0.8} & 1000 & 357.5 & 106.2 & 0.31 & 336.5 & 77.8 & 0.25 \\
\hline & 2000 & 915.8 & 308.8 & 0.42 & 872.2 & 243.3 & 0.36 \\
\hline & 4000 & 1508.4 & 471.8 & 0.33 & 1442.6 & 383.5 & 0.29 \\
\hline & 8000 & 2715.3 & 805.2 & 0.29 & 2575.7 & 615.8 & 0.25 \\
\hline & 16,000 & 7186.8 & 2374.8 & 0.41 & 6829.8 & 1855.2 & 0.35 \\
\hline
\end{tabular}

Table 4

Pegging test (WEAK)

\begin{tabular}{|c|c|c|c|c|c|c|c|}
\hline \multirow[t]{2}{*}{$d$} & \multirow[t]{2}{*}{$n$} & \multicolumn{3}{|l|}{ PLAIN } & \multicolumn{3}{|l|}{ BLOCK } \\
\hline & & $n^{\prime}$ & $m^{\prime}$ & Reduction & $n^{\prime}$ & $m^{\prime}$ & Reduction \\
\hline \multirow[t]{5}{*}{0.1} & 1000 & 210.0 & 7.6 & 0.17 & 207.4 & 5.0 & 0.14 \\
\hline & 2000 & 350.4 & 10.4 & 0.13 & 348.4 & 8.4 & 0.12 \\
\hline & 4000 & 536.2 & 12.0 & 0.09 & 533.1 & 8.9 & 0.08 \\
\hline & 8000 & 2454.6 & 89.8 & 0.26 & 2434.3 & 68.5 & 0.23 \\
\hline & 16,000 & 5680.5 & 211.1 & 0.30 & 5629.1 & 158.1 & 0.26 \\
\hline \multirow[t]{5}{*}{0.2} & 1000 & 270.2 & 19.3 & 0.22 & 263.4 & 12.2 & 0.17 \\
\hline & 2000 & 604.0 & 38.3 & 0.24 & 594.8 & 28.4 & 0.20 \\
\hline & 4000 & 816.2 & 43.7 & 0.15 & 805.0 & 31.6 & 0.13 \\
\hline & 8000 & 3478.3 & 283.0 & 0.39 & 3428.6 & 228.4 & 0.35 \\
\hline & 16,000 & 7282.3 & 592.5 & 0.41 & 7177.4 & 479.1 & 0.37 \\
\hline \multirow[t]{5}{*}{0.4} & 1000 & 328.5 & 46.9 & 0.27 & 316.5 & 33.1 & 0.23 \\
\hline & 2000 & 613.5 & 85.0 & 0.25 & 592.4 & 60.5 & 0.21 \\
\hline & 4000 & 1709.0 & 292.0 & 0.39 & 1666.7 & 240.6 & 0.35 \\
\hline & 8000 & 4372.2 & 714.0 & 0.49 & 4235.1 & 547.7 & 0.43 \\
\hline & 16,000 & 7124.0 & 1143.1 & 0.40 & 6898.1 & 873.3 & 0.34 \\
\hline \multirow[t]{5}{*}{0.8} & 1000 & 464.2 & 140.5 & 0.40 & 433.0 & 99.4 & 0.33 \\
\hline & 2000 & 1017.1 & 327.0 & 0.45 & 963.7 & 250.8 & 0.39 \\
\hline & 4000 & 2585.4 & 935.7 & 0.61 & 2473.7 & 764.5 & 0.54 \\
\hline & 8000 & 4619.7 & 1628.9 & 0.54 & 4424.0 & 1337.2 & 0.48 \\
\hline & 16,000 & $14,289.6$ & 5414.3 & 0.87 & $13,838.7$ & 4729.6 & 0.80 \\
\hline
\end{tabular}


The second observation above may better be explained in terms of the close relation between the average gap (in Tables 1 and 2) and the ineffectiveness of pegging measured by 'reduc', which can be represented as the following regression functions:

$$
\begin{aligned}
& \text { UNCOR }: \text { reduc }=0.015+0.00097 \cdot \text { gap } \quad\left(R^{2}=0.970\right) \\
& \text { WEAK }: \text { reduc }=0.153+0.00230 \cdot \operatorname{gap} \quad\left(R^{2}=0.815\right)
\end{aligned}
$$

For example, if we have gap $=100$, the expected ratio of reduction would be 0.11 in UNCOR, and 0.38 in WEAK.

Table 5

\begin{tabular}{|c|c|c|c|c|c|c|c|c|}
\hline \multirow[t]{2}{*}{$d$} & \multirow[t]{2}{*}{$n$} & \multirow[t]{2}{*}{ \#sol } & \multicolumn{3}{|c|}{ CPU seconds } & \multicolumn{3}{|l|}{$\mathrm{BBN}$} \\
\hline & & & Average & Maximum & Minimum & Average & Maximum & Minimum \\
\hline \multirow[t]{5}{*}{0.1} & 1000 & 10 & 6.92 & 14.26 & 0.63 & 1185.7 & 6688 & 103 \\
\hline & 2000 & 10 & 22.60 & 80.03 & 0.65 & 3067.5 & 11,966 & 14 \\
\hline & 4000 & 7 & 75.58 & 180.99 & 14.84 & 5196.1 & 12,888 & 653 \\
\hline & 8000 & 7 & 88.47 & 243.79 & 12.46 & 1601.2 & 5206 & 27 \\
\hline & 16,000 & 5 & 425.29 & 1011.85 & 29.32 & 5101.2 & 16,138 & 24 \\
\hline \multirow[t]{5}{*}{0.2} & 1000 & 10 & 8.44 & 19.84 & 0.46 & 1966.1 & 4529 & 54 \\
\hline & 2000 & 10 & 18.47 & 71.29 & 2.94 & 2196.6 & 9426 & 319 \\
\hline & 4000 & 6 & 43.17 & 71.54 & 22.10 & 1681.8 & 3878 & 392 \\
\hline & 8000 & 7 & 200.10 & 573.94 & 38.60 & 4086.0 & 18,629 & 230 \\
\hline & 16,000 & 3 & 284.11 & 631.02 & 52.82 & 998.3 & 2364 & 18 \\
\hline \multirow[t]{5}{*}{0.4} & 1000 & 10 & 11.41 & 40.53 & 1.37 & 1966.5 & 7303 & 251 \\
\hline & 2000 & 10 & 27.91 & 89.07 & 3.10 & 2116.4 & 8330 & 144 \\
\hline & 4000 & 6 & 82.81 & 159.76 & 10.73 & 2247.5 & 4318 & 280 \\
\hline & 8000 & 6 & 426.55 & 1853.22 & 15.72 & $23,800.8$ & 129,326 & 55 \\
\hline & 16,000 & 3 & 437.57 & 526.79 & 384.81 & 1210.7 & 1867 & 672 \\
\hline \multirow[t]{5}{*}{0.8} & 1000 & 10 & 16.47 & 34.96 & 2.62 & 1930.0 & 3521 & 302 \\
\hline & 2000 & 10 & 91.52 & 176.58 & 4.30 & 3520.0 & 6310 & 167 \\
\hline & 4000 & 7 & 142.64 & 300.57 & 52.84 & 1984.4 & 3680 & 673 \\
\hline & 8000 & 3 & 180.13 & 266.85 & 27.68 & 1248.0 & 2611 & 38 \\
\hline & 16,000 & 3 & 549.27 & 879.44 & 190.37 & 1475.3 & 2869 & 110 \\
\hline
\end{tabular}

Exact solutions by NUOPT (UNCOR)

Table 6

\begin{tabular}{|c|c|c|c|c|c|c|c|c|}
\hline \multirow[t]{2}{*}{$d$} & \multirow[t]{2}{*}{$n$} & \multirow[t]{2}{*}{ \#sol } & \multicolumn{3}{|c|}{ CPU seconds } & \multicolumn{3}{|l|}{$\mathrm{BBN}$} \\
\hline & & & Average & Maximum & Minimum & Average & Maximum & Minimum \\
\hline \multirow[t]{3}{*}{0.1} & 1000 & 10 & 46.87 & 207.20 & 1.99 & $27,310.9$ & 117,046 & 775 \\
\hline & 2000 & 7 & 678.33 & 2739.03 & 1.42 & $255,172.3$ & $1,055,088$ & 40 \\
\hline & 4000 & 5 & 757.12 & 1832.44 & 15.78 & $143,128.8$ & 373,478 & 366 \\
\hline \multirow[t]{3}{*}{0.2} & 1000 & 10 & 45.06 & 172.12 & 1.19 & $24,920.5$ & 101,854 & 210 \\
\hline & 2000 & 7 & 325.08 & 937.88 & 8.95 & $106,151.4$ & 321,047 & 605 \\
\hline & 4000 & 3 & 6.95 & 8.39 & 4.23 & 51.7 & 84 & 17 \\
\hline \multirow[t]{3}{*}{0.4} & 1000 & 10 & 37.89 & 199.45 & 3.63 & $16,031.0$ & 103,943 & 1116 \\
\hline & 2000 & 10 & 338.39 & 1831.27 & 2.66 & $117,147.8$ & 692,331 & 94 \\
\hline & 4000 & 2 & 348.10 & 395.39 & 300.81 & $40,286.5$ & 44,968 & 35,605 \\
\hline \multirow[t]{3}{*}{0.8} & 1000 & 10 & 36.16 & 162.49 & 0.44 & $11,402.0$ & 71,155 & 185 \\
\hline & 2000 & 8 & 276.46 & 1039.92 & 7.13 & $64,435.5$ & 271,908 & 360 \\
\hline & 4000 & 5 & 311.57 & 840.26 & 68.72 & $33,628.2$ & 124,502 & 1085 \\
\hline
\end{tabular}

Exact solutions by NUOPT (WEAK) 


\subsection{Exact solutions}

We solve the problems to optimality using NUOPT [20] directly, as well as by the block pegging (referred to as BLOCK) and the virtual pegging methods (VIRTUAL). NUOPT is a commercial MP solver developed by a Japanese vendor, and is claimed to be competitive to such popular solvers as LINDO or CPLEX. We use NUOPT to solve the reduced problems in BLOCK and VIRTUAL as well. Tables 5 and 6 show the results of NUOPT, Tables 7 and 8 are those of BLOCK, and Tables 9 and 10 summarize the results of VIRTUAL, all for UNCOR and WEAK cases.

For each value of $d$ and $n$, the same 10 instances were solved as in Tables 1 and 2 with the limit time of 3600 CPU seconds. Here \#sol denotes the number of solved instances within this time limit, and \#rep in Tables 9 and 10 is the number of repetitions of Steps 2-4 in Virtual_Pegging Test. In these tables 'CPU seconds' is the sum of the computation time for the upper and lower bounds, pegging test, and the time to solve the reduced problem using NUOPT. Also 'BBN' is the number of the branch and bound nodes generated by NUOPT to solve respective problems. The average, maximum and minimum of these values over the solved (\#sol) instances are given in these tables.

In VIRTUAL we fixed $\delta$ at $\delta=10$, since the average of the gap $\bar{z}-z^{\star}$ was approximately 10 in the computation of BLOCK. We observe the followings

1. BLOCK often solves instances that NUOPT cannot solve, and VIRTUAL sometimes solves instances that BLOCK cannot. The converse is very rare. In our experiments, NUOPT was able to solve only one instance (WEAK, $d=0.2, n=2000$ ) that BLOCK could not solve. This instance was solved by VIRTUAL.

2. In UNCOR cases, NUOPT frequently fails to solve instances with $n \geqslant 4000$. Contrary, for $n \leqslant 8000$ almost all instances were solved either by BLOCK or VIRTUAL. Even if NUOPT solves these instances, it is usually time consuming.

3. Correlation between weights and profits makes problems much harder. In WEAK, NUOPT sometimes fails for problems with $n=2000$. With VIRTUAL we are able to solve these problems, but compared to the UNCOR instances, it takes much longer CPU time.

Table 7

Exact solutions by BLOCK (UNCOR)

\begin{tabular}{|c|c|c|c|c|c|c|c|c|}
\hline \multirow[t]{2}{*}{$d$} & \multirow[t]{2}{*}{$n$} & \multirow[t]{2}{*}{ \#sol } & \multicolumn{3}{|c|}{ CPU seconds } & \multicolumn{3}{|l|}{$\mathrm{BBN}$} \\
\hline & & & Average & Maximum & Minimum & Average & Maximum & Minimum \\
\hline \multirow[t]{5}{*}{0.1} & 1000 & 10 & 0.72 & 2.53 & 0.19 & 662.5 & 2555 & 92 \\
\hline & 2000 & 10 & 1.38 & 3.33 & 0.64 & 1183.4 & 2953 & 14 \\
\hline & 4000 & 10 & 9.18 & 19.83 & 2.85 & 8999.8 & 30,966 & 612 \\
\hline & 8000 & 10 & 292.40 & 2753.70 & 7.50 & $278,314.1$ & $2,622,402$ & 29 \\
\hline & 16,000 & 8 & 244.28 & 1223.88 & 25.57 & $217,718.9$ & $1,128,487$ & 20 \\
\hline \multirow[t]{5}{*}{0.2} & 1000 & 10 & 0.84 & 1.78 & 0.38 & 866.0 & 2007 & 54 \\
\hline & 2000 & 10 & 1.74 & 4.27 & 0.69 & 1492.6 & 2491 & 181 \\
\hline & 4000 & 10 & 17.62 & 102.24 & 3.66 & $22,353.2$ & 169,373 & 1015 \\
\hline & 8000 & 10 & 254.35 & 2366.15 & 7.39 & $480,265.1$ & $4,744,547$ & 239 \\
\hline & 16,000 & 8 & 240.36 & 827.42 & 33.09 & $184,046.6$ & 539,396 & 18 \\
\hline \multirow[t]{5}{*}{0.4} & 1000 & 10 & 1.41 & 3.21 & 0.51 & 906.2 & 2627 & 244 \\
\hline & 2000 & 10 & 11.25 & 49.88 & 1.30 & 1530.9 & 8100 & 22 \\
\hline & 4000 & 9 & 11.39 & 31.84 & 4.42 & 2228.0 & 4714 & 270 \\
\hline & 8000 & 8 & 67.38 & 238.14 & 24.69 & $280,91.9$ & 137,069 & 54 \\
\hline & 16,000 & 6 & 382.49 & 1292.05 & 111.70 & $551,550.0$ & $2,744,246$ & 667 \\
\hline \multirow[t]{5}{*}{0.8} & 1000 & 10 & 4.77 & 24.52 & 1.09 & 1379.6 & 4489 & 302 \\
\hline & 2000 & 10 & 43.16 & 145.19 & 3.83 & 2611.3 & 6525 & 167 \\
\hline & 4000 & 10 & 68.44 & 165.79 & 27.89 & 4269.1 & 13,730 & 674 \\
\hline & 8000 & 6 & 138.74 & 340.02 & 53.82 & $15,895.3$ & 72,327 & 38 \\
\hline & 16,000 & 8 & 784.37 & 2782.35 & 290.37 & $48,035.8$ & 296,551 & 92 \\
\hline
\end{tabular}


Table 8

Exact solutions by BLOCK (WEAK)

\begin{tabular}{|c|c|c|c|c|c|c|c|c|}
\hline \multirow[t]{2}{*}{$d$} & \multirow[t]{2}{*}{$n$} & \multirow[t]{2}{*}{ \#sol } & \multicolumn{3}{|c|}{ CPU seconds } & \multicolumn{3}{|l|}{$\mathrm{BBN}$} \\
\hline & & & Average & Maximum & Minimum & Average & Maximum & Minimum \\
\hline \multirow[t]{3}{*}{0.1} & 1000 & 10 & 13.49 & 88.28 & 0.80 & $20,105.0$ & 112,990 & 783 \\
\hline & 2000 & 8 & 235.46 & 978.73 & 0.71 & $467,400.3$ & $1,972,277$ & 40 \\
\hline & 4000 & 8 & 370.14 & 1498.35 & 1.66 & $510,953.8$ & $2,635,522$ & 343 \\
\hline \multirow[t]{3}{*}{0.2} & 1000 & 10 & 14.11 & 61.45 & 0.57 & $23,210.9$ & 99,723 & 210 \\
\hline & 2000 & 8 & 261.94 & 1589.88 & 0.69 & $323,718.1$ & $1,961,311$ & 615 \\
\hline & 4000 & 5 & 674.36 & 1899.56 & 1.91 & $648,243.0$ & $1,690,114$ & 27 \\
\hline \multirow[t]{3}{*}{0.4} & 1000 & 10 & 10.06 & 44.83 & 1.67 & $16,158.7$ & 99,723 & 1265 \\
\hline & 2000 & 10 & 112.29 & 516.55 & 1.84 & $118,568.5$ & 692,357 & 99 \\
\hline & 4000 & 3 & 301.02 & 843.68 & 2.78 & $763,563.0$ & $2,249,335$ & 8407 \\
\hline \multirow[t]{3}{*}{0.8} & 1000 & 10 & 15.29 & 66.83 & 1.18 & $11,355.2$ & 68,881 & 1 \\
\hline & 2000 & 10 & 131.78 & 1008.87 & 9.36 & $44,449.5$ & 272,364 & 378 \\
\hline & 4000 & 6 & 437.26 & 1228.72 & 9.98 & $397,797.8$ & $2,178,465$ & 1159 \\
\hline
\end{tabular}

Table 9

Exact solutions by VIRTUAL (UNCOR)

\begin{tabular}{|c|c|c|c|c|c|c|c|c|c|}
\hline \multirow[t]{2}{*}{$\bar{d}$} & \multirow[t]{2}{*}{$n$} & \multirow[t]{2}{*}{ \#sol } & \multirow[t]{2}{*}{ \#rep } & \multicolumn{3}{|c|}{ CPU seconds } & \multicolumn{3}{|l|}{$\mathrm{BBN}$} \\
\hline & & & & Average & Maximum & Minimum & Average & Maximum & Minimum \\
\hline \multirow[t]{5}{*}{0.1} & 1000 & 10 & 1.5 & 1.04 & 1.56 & 0.11 & 229.5 & 484 & 31 \\
\hline & 2000 & 10 & 1.0 & 0.88 & 1.95 & 0.50 & 1339.1 & 6073 & 14 \\
\hline & 4000 & 10 & 1.0 & 4.36 & 11.15 & 1.83 & 6475.4 & 26,673 & 417 \\
\hline & 8000 & 10 & 1.0 & 130.44 & 1216.76 & 4.07 & $258,468.1$ & $2,491,418$ & 29 \\
\hline & 16,000 & 8 & 1.0 & 151.18 & 737.19 & 10.80 & $215,314.9$ & $1,135,948$ & 22 \\
\hline \multirow[t]{5}{*}{0.2} & 1000 & 10 & 1.4 & 0.47 & 0.88 & 0.20 & 286.7 & 656 & 54 \\
\hline & 2000 & 10 & 1.2 & 1.56 & 2.48 & 0.62 & 1117.3 & 5369 & 172 \\
\hline & 4000 & 10 & 1.0 & 4.30 & 7.79 & 2.22 & 5212.5 & 16,227 & 542 \\
\hline & 8000 & 10 & 1.0 & 210.14 & 1992.21 & 5.54 & $47,9791.7$ & $4,746,000$ & 230 \\
\hline & 16,000 & 8 & 1.0 & 129.66 & 313.95 & 20.53 & $169,248.8$ & 483,664 & 18 \\
\hline \multirow[t]{5}{*}{0.4} & 1000 & 10 & 1.4 & 0.65 & 1.46 & 0.32 & 289.8 & 649 & 78 \\
\hline & 2000 & 10 & 3.6 & 18.26 & 27.55 & 0.82 & 870.4 & 4244 & 128 \\
\hline & 4000 & 10 & 1.0 & 8.46 & 17.18 & 3.43 & 1815.1 & 3614 & 162 \\
\hline & 8000 & 10 & 3.8 & 293.86 & 788.74 & 17.50 & $275,61.6$ & 147,355 & 54 \\
\hline & 16,000 & 7 & 1.9 & 438.59 & 2189.79 & 56.22 & $594,641.6$ & $3,509,838$ & 58 \\
\hline \multirow[t]{5}{*}{0.8} & 1000 & 10 & 5.0 & 3.53 & 5.65 & 0.64 & 313.3 & 946 & 25 \\
\hline & 2000 & 10 & 4.0 & 11.72 & 23.79 & 3.46 & 608.9 & 1204 & 350 \\
\hline & 4000 & 10 & 4.0 & 68.64 & 85.94 & 19.30 & 2471.7 & 12,999 & 22 \\
\hline & 8000 & 9 & 1.1 & 77.25 & 120.58 & 50.89 & 8280.4 & 25,000 & 38 \\
\hline & 16,000 & 10 & 1.9 & 608.24 & 848.37 & 241.89 & $123,548.5$ & 470,867 & 1228 \\
\hline
\end{tabular}

One reason of the poor performance in WEAK instances is that we are using NUOPT to solve the reduced problem. Even for standard KPs the correlated instances are often hard to solve by general purpose MP solvers.

\subsection{Sensitivity analysis}

Table 11 gives the result of a sensitivity analysis with respect to the knapsack capacity. We fix $d=0.2$, and consider the UNCOR case. We compare the CPU time in seconds, with average, maximum and minimum over 
Table 10

Exact solutions by VIRTUAL (WEAK)

\begin{tabular}{|c|c|c|c|c|c|c|c|c|c|}
\hline \multirow[t]{2}{*}{$d$} & \multirow[t]{2}{*}{$n$} & \multirow[t]{2}{*}{ \#sol } & \multirow[t]{2}{*}{ \#rep } & \multicolumn{3}{|c|}{ CPU seconds } & \multicolumn{3}{|l|}{$\mathrm{BBN}$} \\
\hline & & & & Average & Maximum & Minimum & Average & Maximum & Minimum \\
\hline \multirow[t]{3}{*}{0.1} & 1000 & 10 & 1.0 & 9.52 & 63.69 & 0.55 & $23,096.1$ & 147,336 & 783 \\
\hline & 2000 & 10 & 1.0 & 591.24 & 1282.07 & 0.77 & $288,462.3$ & $1,067,322$ & 40 \\
\hline & 4000 & 8 & 1.0 & 396.01 & 1912.41 & 1.74 & $585,500.0$ & $2,789,513$ & 348 \\
\hline \multirow[t]{3}{*}{0.2} & 1000 & 10 & 1.0 & 9.87 & 39.65 & 0.44 & $25,249.7$ & 98,486 & 176 \\
\hline & 2000 & 9 & 1.0 & 349.69 & 1716.75 & 0.89 & $698,107.2$ & $3,673,265$ & 596 \\
\hline & 4000 & 6 & 1.0 & 827.03 & 2765.84 & 1.99 & $1,113,609.0$ & $3,692,637$ & 25 \\
\hline \multirow[t]{3}{*}{0.4} & 1000 & 10 & 1.0 & 6.79 & 43.14 & 0.99 & $15,299.0$ & 100,793 & 1151 \\
\hline & 2000 & 10 & 1.0 & 63.63 & 317.41 & 1.58 & $130,041.8$ & 692,126 & 96 \\
\hline & 4000 & 5 & 1.0 & 1080.53 & 2532.03 & 11.71 & $1,382,056.0$ & $3,113,746$ & 318 \\
\hline \multirow[t]{3}{*}{0.8} & 1000 & 10 & 1.0 & 4.52 & 30.14 & 0.98 & 7435.6 & 65,397 & 1 \\
\hline & 2000 & 10 & 1.2 & 29.97 & 163.93 & 7.07 & $420,83.8$ & 270,575 & 380 \\
\hline & 4000 & 8 & 1.1 & 1065.24 & 3156.37 & 11.91 & $1,322,136.0$ & $4,131,502$ & 1817 \\
\hline
\end{tabular}

Table 11

Sensitivity of the CPU time to the knapsack capacity

\begin{tabular}{|c|c|c|c|c|c|c|c|c|c|}
\hline \multirow[t]{2}{*}{$n$} & \multicolumn{3}{|l|}{$c=125 n$} & \multicolumn{3}{|l|}{$c=250 n$} & \multicolumn{3}{|l|}{$c=375 n$} \\
\hline & Minimum & Average & Maximum & Minimum & Average & Maximum & Minimum & Average & Maximum \\
\hline 1000 & 0.24 & 0.70 & 1.35 & 0.38 & 0.85 & 1.83 & 0.12 & 0.39 & 1.34 \\
\hline 2000 & 0.83 & 1.70 & 3.54 & 0.70 & 1.95 & 4.17 & 0.36 & 0.89 & 1.35 \\
\hline 4000 & 2.92 & 5.10 & 8.47 & 3.61 & 18.31 & 101.76 & 1.70 & 3.49 & 7.27 \\
\hline 8000 & 13.05 & 21.53 & 44.92 & 7.39 & 280.67 & 2452.00 & 7.58 & 15.99 & 59.46 \\
\hline
\end{tabular}

Table 12

CPU seconds for strongly correlated instances

\begin{tabular}{|c|c|c|c|c|c|c|c|c|c|}
\hline \multirow[t]{2}{*}{$n$} & \multirow[t]{2}{*}{$m$} & \multicolumn{2}{|c|}{ NUOPT } & \multicolumn{6}{|c|}{ BLOCK } \\
\hline & & $\mathrm{CPU}$ & \#sol & gap & $n^{\prime}$ & $m^{\prime}$ & Reduction & CPU & \#sol \\
\hline 100 & 9.1 & 93.3 & 9 & 48.9 & 58.3 & 3.2 & 45.0 & 89.2 & 9 \\
\hline 200 & 21.1 & 455.5 & 5 & 55.1 & 119.5 & 8.5 & 47.4 & 182.5 & 6 \\
\hline
\end{tabular}

10 random instances shown in the table for the cases of $c=125 n, c=250 n$ and $c=375 n$. These represent approximately $1 / 4,1 / 2$ and $3 / 4$ of the total weight of items.

From the table we see that PCKP is most difficult when the knapsack capacity is near to $1 / 2$ of the total weight of items, while the problem is easier to solve for the knapsack capacity far apart from this value.

Finally, we solved some 'strongly correlated' instances, where $w_{j}$ is uniformly distributed over $[1,1000]$, but $p_{j}$ is related to $w_{j}$ by

$$
p_{j}=w_{j}+200 .
$$

We show a result in Table 12. As in ordinary knapsack problems, this type of PCKP is quite difficult to solve. This is because the weakness of the solvers to solve strongly correlated knapsack problems. We were unable to solve PCKPs with $n \geqslant 400$ by any of the methods considered in our work.

\section{Conclusion}

In this paper, we have shown that the pegging test for the standard 0-1 KPs can be extended to PCKPs by introducing the Lagrangian relaxation first to the precedence constraints. In addition, by proposing the block and virtual pegging tests, we were able to solve PCKPs with up to 16,000 items. 
However, the experiments in this paper were limited to PCKPs with 'sparse' precedence relations in the sense that $|E|=O(n)$. Also, these relations were randomly picked up from all the possible pairs. For instances with 'dense' precedence relations such as $|E|=O\left(n^{2}\right)$, or with $E$ generated from some other random mechanisms, the results may be quite different. These 'instance characteristics' of the algorithm are left for future investigations.

To solve yet larger problems exactly we need to explore the methods to obtain more tight upper and lower bounds, as well as the specialized algorithms to solve the reduced problem more efficiently. Analysis of polyhedral structure of PCKP $[4,3]$ may prove useful in this direction.

\section{Acknowledgements}

The authors express their appreciation to Mr. S. Aminto (currently with the Indonesian Air Force) for his help in some part of the computation. They are also greatful to anonymous referees for informing us of a polyhedral analysis reference, as well as for their constructive comments.

\section{References}

[1] E. Aarts, J.K. Lenstra (Eds.), Local Search in Combinatorial Optimization, John Wiley \& Sons, Chichester, 1997.

[2] R.K. Ahuja, T.L. Magnanti, J.B. Orlin, Network Flows: Theory, Algorithms, and Applications, Prentice Hall, Englewood Criffs, 1993.

[3] N. Boland, C. Fricke, G. Floyland, R. Sotirov, Clique-based facets for the precedence constrained knapsack problem, Optimization Online. http://www.optimization-online.org/DB_HTML/01/1322.html, 2006.

[4] E.A. Boyd, Polyhedral results for the precedence-constrained knapsack problem, Discrete Applied Mathematics 41 (1993) $185-201$.

[5] G. Cho, D.X. Shaw, A depth-first dynamic programming algorithm for the tree knapsack problem, INFORMS Journal on Computing 9 (1997) 431-438.

[6] R.S. Dembo, P.L. Hammer, A reduction algorithm for knapsack problems, Methods of Operations Research 36 (1980) 49-60.

[7] D. Fayard, G. Plateau, Resolution of the 0-1 knapsack problem: Comparison of methods, Mathematical Programming 8 (1975) 272307.

[8] R. Fourer, Software survey: Linear programming, OR/MS Today 26 (1999) 64-71.

[9] M.R. Garey, D.S. Johnson, Computers and Intractability: A Guide to the Theory of NP-Completeness, Freeman and Company, New York, 1979.

[10] F. Harary, Graph Theory, Addison Wesley, Reading, 1969.

[11] R. Hirabayashi, H. Suzuki, N. Tuchiya, Optimal tool module design problem for NC machine tools, Journal of the Operations Research Society of Japan 27 (1983) 205-229.

[12] D.D. Hochbaum, Selection, provisioning, shared fixed costs, maximum closure, and implications on algorithmic methods today, Management Science 50 (2004) 709-723.

[13] G.P. Ingargiola, J.F. Korsh, A reduction algorithm for zero-one single knapsack problems, Management Science 20 (1973) $460-463$.

[14] D.S. Johnson, K.A. Niemi, On knapsacks, partitions, and a new dynamic programming technique for trees, Mathematics of Operations Research 8 (1983) 1-14.

[15] H. Kellerer, U. Pferschy, D. Pisinger, Knapsack Problems, Springer Verlag, 2004.

[16] T. Kuno, H. Konno, E. Zemel, A linear-time algorithm for solving continuous maximin knapsack problems, Operations Research Letters 10 (1991) 23-26.

[17] S. Martello, P. Toth, Knapsack Problems: Algorithms and Computer Implementations, Wiley, New York, 1990.

[18] H. Moriyama, T. Hada, H. Suzuki, A partially ordered knapsack problem and scheduling, in: Proceedings of the Production Scheduling Symposium'96, Japan Industrial Management Association, 1996, pp. 79-84 (in Japanese).

[19] G.L. Nemhauser, L.A. Wolsey, Integer and Combinatorial Optimization, John Wiley \& Sons, New York, 1988.

[20] Mathematical Systems Incorporated (in Japanese). NUOPT manual. http://www.msi.co.jp/nuopt, 2002.

[21] N. Samphaiboon, T. Yamada, Heuristic and exact algorithms for the precedence-constrained knapsack problem, Journal of Optimization Theory and Application 105 (2002) 659-676.

[22] D.X. Shaw, G. Cho, The critical-item, upper bounds, and a branch-and-bound algorithm for the tree knapsack problem, Networks 28 (1998) 205-216.

[23] L.A. Wolsey, Integer Programming, John Wiley \&Sons, New York, 1998.

[24] T. Yamada. http://www.nda.ac.jp/ yamada/ypublication.html, 2006. 